\title{
Feedback control of ECRH polarization on LHD
}

\author{
F. Felici ${ }^{1}$, T. Shimozuma ${ }^{2}$, S. Kubo ${ }^{2}$, Y. Yoshimura ${ }^{2}$, \\ H. Takahashi ${ }^{2}$, H. Igami ${ }^{2}$, T.P. Goodman ${ }^{1}$, T. Seki ${ }^{2}$, \\ H. Tsuchiya ${ }^{2}$, S. Ito ${ }^{2}$, Y. Mizuno ${ }^{2}$ O. Sauter ${ }^{1}$, T. Mutoh ${ }^{2}$ and \\ the LHD Experiment Group
}

${ }^{1}$ École Polytechnique Fédérale de Lausanne (EPFL)

Centre de Recherches en Physique des Plasmas

Association EURATOM-Suisse

1015 Lausanne, Switzerland

${ }^{2}$ National Institite for Fusion Science (NIFS)

Oroshi-cho, Toki 509-5292, Japan

E-mail: federico.felici@epfl.ch

\begin{abstract}
The polarization of ECRH waves, set by the orientation of a pair of corrugated mirror polarizers in the transmission line, determines the degree of coupling to $\mathrm{O}-$ and $\mathrm{X}$-modes in the plasma and has an important effect on the first-pass absorption. Existing methods for determining the required polarization have been found adequate in most experiments. However, as the pulse length is increased it becomes increasingly important to maximize the first-pass absorption while the plasma or injection conditions change or when there can be significant O- to Xmode power coupling during propagation, particularly in the edge plasma region of a stellarator. This has motivated the development of a dedicated feedback control system which is able to adjust the polarizers' angles settings during the discharge in order to maintain the highest possible absorption. An extremum seeking controller is shown to successfully recover the optimum polarization setting during long-pulse ECRH experiments on LHD.
\end{abstract}

PACS numbers: 07.05.Dz,52.25.Os,52.35.Mw,52.50.-b,52.50.Sw,52.55.Hc,52.55.Fa

Submitted to: Nuclear Fusion

August 23, 2010

\section{Introduction}

As the pulse length achieved by magnetic confinement fusion experiments becomes longer, demands on auxiliary heating systems become increasingly stringent, in particular concerning the demand that the power should be deposited efficiently in the plasma in order to minimize the amount of stray power reaching and possibly damaging in-vessel components and diagnostics. Electron Cyclotron Resonance Heating and Current Drive (ECRH/ECCD) have been successful as experimental heating and 
current drive methods for diverse applications such as the suppression of MHD modes, confinement studies and also as a bulk plasma heating method and is envisaged on future devices [1].

The Large Helical Device (LHD) [2] is the largest heliotron in the world, having $l=2 / m=10$ superconducting helical windings with major radius $R_{0}=3.9 \mathrm{~m}$ and minor radius $a=0.6 \mathrm{~m}$. Its auxiliary heating systems include NBI, ICRH and ECRH. The ECRH system is composed of several gyrotrons at different frequencies [3] and is used for plasma production, long pulse plasma sustainment [4], and for other physics studies [5], 6]. In particular, the system includes three $77 \mathrm{GHz}$ gyrotrons capable of $1.5 \mathrm{MW}$ for 2 seconds and 330kW during 420s which is almost Continuous Wave (CW) [7]. They are connected to the vacuum vessel via evacuated transmission lines compatible with CW operation. As pulse lengths of several minutes are achieved with this system, optimizing the degree of first-pass absorption is of great importance since the total energy deposited on in-vessel components may become significant in the case of nonnegligible shine-through or multiple reflections of the injected ECRH power. This will also be of great importance in future long-pulse devices such as W7-X [8], 9] and ITER [10], [11] and is a topic of research on LHD.

\subsection{Polarization settings for EC waves in fusion devices}

In typical ECRH injection scenarios, one of the two possible wave modes is desired in the plasma. When first harmonic heating is envisaged, one typically requires O-mode as the $\mathrm{X}$-mode has a cut-off before the resonance layer along the ray path. Second harmonic heating scenarios tend to use X-mode, as it is well absorbed while the second harmonic O-mode has less favorable absorption (except at high densities where the X-mode is cut off). As is well known, the degree to which the free-space propagating EC wave couples to $\mathrm{O}$ - or $\mathrm{X}$-modes depends on the polarization of the wave in relation to the relative orientation of the magnetic field vector and the wave propagation vector at, or near, the last closed flux surface (LCFS) [12]. On existing devices, the orientation of the magnetic field vector at this point is known from the plasma equilibrium, and the orientation of the wave propagation vector can be inferred from the knowledge of the launching antenna position and orientation. From this, the required polarization of the free-space EC wave at the interface for either O- or X-mode can be calculated [13]. Polarization is parameterized by the polarization rotation angle $\left(-90^{\circ} \leq \alpha \leq 90\right)$ and the degree of ellipticity $\left(-45^{\circ} \leq \beta \leq 45^{\circ}\right)$, see Figure 1. Depending on the mode ( $\mathrm{O}$ or $\left.\mathrm{X}\right)$, the plasma properties and the injection geometry, the required polarization can be very different for different scenarios, often requiring a combination of $\alpha$ and $\beta$ for complete coupling for current drive injection. ECRH transmission lines are therefore equipped with devices to change the polarization of the EC wave, typically in the form of two corrugated mirrors in high power applications [14], [15], although several novel methods can be used [16]. In general, two corrugated mirrors with groove depth in the order of $1 / 4$ or $1 / 8$ of the free space wavelength $\lambda$ can be used to vary the polarization rotation 
angle $(\alpha)$ and the ellipticity $(\beta)$, respectively. The obtained polarization depends on the polarizer angles $\left(\phi_{\lambda / 4}, \phi_{\lambda / 8}\right)$, defined as the orientation of the grooves with respect to the plane of incidence for the polarizing mirror with groove depth of order $\lambda / 4$ and $\lambda / 8$, respectively. With knowledge of the polarizer mirror characteristics and the geometry of all transmission line components, it is possible to rapidly calculate the polarization of the EC wave at an arbitrary point along the beam path, including the intersection with the LCFS. A numerical search routine is then typically used to determine a combination of polarizer angles which yields the values of $\alpha, \beta$ required for $100 \%$ coupling to a particular mode in the plasma [17]. It should be noted that analogous issues arise when performing ECE measurements, since it is usually required that only one mode ( $\mathrm{O}$ or $\mathrm{X}$ ) of the EC radiation exiting the plasma is coupled to the ECE receiver.

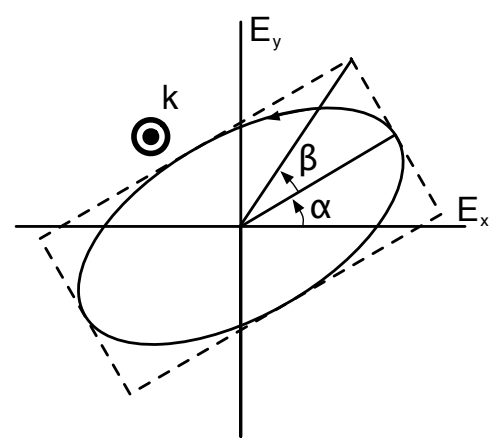

Figure 1: Definitions of polarization angles $\alpha$ and $\beta$ relative to the ellipse swept by the wave electric field vector.

\subsection{Motivations for feedback control system}

There are several reasons why the pre-calculated polarizer settings may not be entirely accurate, particularly in heliotrons. In LHD it was found that the pre-calculated polarization does not always correspond to the experimentally obtained optimum [18]. One reason for inaccuracy stems from imprecise knowledge of the LCFS location, particularly in regions with ergodized field lines. Moreover, in regions with gradual density gradients [19] the LCFS may not be the ideal surface on which to base the coupling calculations. Another origin of the inaccuracy is due to the coupling between Oand X-mode which may be important in regions of significant magnetic shear, depending on the density and density gradients. This effect was studied in [20] and [21], and was seen as an important effect in ECRH polarization optimization for ATF [22], HeliotronE [23], CHS [24], LHD [25], [18] and is also significant for ECE measurements on LHD [26].

Even if the required polarization setting is known perfectly by taking all the relevant 
physics effects into account, it may be necessary to adjust the polarization of the EC wave during the shot if the plasma conditions change. Several factors may contribute to a change of optimal coupling, such as changes in the plasma current in a tokamak discharge, change of shape of the last closed flux surface, change of plasma position, and change in gyrotron frequency in the case of multifrequency gyrotrons. For example, changes in the plasma current during a tokamak discharge lead to different contributions of the edge poloidal field and thus to changes in the $B$-field orientation. Also, changes in the density may alter the degree of power coupling between $\mathrm{O}$ and $\mathrm{X}$-modes in the sheared field configuration in a heliotron such as LHD, requiring adjustment of the polarization to maintain optimal coupling. Finally, changes in the ray path while changing the launcher injection of the ITER upper and equatorial ECRH Launchers [10] [11] will require corresponding changes to the polarizer settings.

One may attempt to pre-calculate and account for some of these effects, but it remains difficult to diagnose (especially in real-time). Ultimately, proof of the adequate polarization settings can only come from a measurement of the coupling to the plasma itself. In practice, one needs a method to answer the question whether the current polarizer settings are the optimal ones yielding maximum first-pass absorption. Dedicated experiments, scanning the polarizer angle settings for different plasma and injection scenarios, can provide part of the answer but are lengthy to complete and may ultimately not cover all the configurations required by the experimental program.

This has motivated the development of a feedback control system for the polarization of the EC waves. If the duration of the plasma exceeds by far the typical rotation time of the polarizers, one can attempt to optimize the polarizer settings during the plasma discharge itself to either obtain a setting with higher power coupling than the pre-calculated setting, or verify that no better setting exists. Additionally, such a feedback control system should be able to track the optimal polarization settings during evolution of the plasma or changes in the ECRH injection geometry. The design and testing of such a feedback control system on LHD is the topic of the remainder of this paper.

The paper is structured as follows. Section 2 is dedicated to the choice and design of the feedback controller, its testing in simulations, and also includes a brief discussion of various diagnostic options for measuring the non-absorbed power. Section 3 describes the experimental set-up and plasma scenario used. Section 4 contains the experimental results, which are subsequently discussed in section 5 .

\section{Feedback controller design}

In control terms, the problem of optimizing the polarizer settings in real-time can be formulated as the problem of finding, in real-time, an optimum (extremum) of a signal indicating the first-pass absorption. At steady state, after effects on the plasma response time scale have subsided, the first-pass absorption can be described by a static map of the polarizer angles $\left(\phi_{\lambda / 8}, \phi_{\lambda / 4}\right)$ to the mode purity which has a local maximum at points 
corresponding to either pure O- or pure X-mode, depending on the plasma scenario. The task of the controller is to steer the polarizer angles from a potentially non-optimal setting to an optimal setting (local maximum) in the least possible time. This situation differs from the majority of control problems encountered in the engineering literature because the sign of the error is not known, i.e. the controller does not know a-priori in what direction to move to improve the situation.

Several possibilities exist in the literature to tackle these kind of problems. The simplest is the use of a procedure borrowed from the numerical optimization literature, for example hill-climbing algorithms such as a cyclic coordinate search [27. These algorithms optimize one variable at a time by (i) searching in the two possible directions along the given variable (ii) determining the direction of positive gradient, and (iii) moving in this direction until a local maximum is reached. The procedure (i)-(iii) is cyclically repeated for each coordinate, i.e. for the two polarizer angles. During an exploratory phase of the feedback controller design, this type of algorithm was implemented and tested in a low-power test stand [28]. A type of feedback controller, more appropriate for real-time implementation than the hillclimbing numerical algorithm mentioned earlier, is the so-called extremum seeking controller. This is a type of nonlinear adaptive controller which has been used in other applications in the magnetic confinement fusion community: control of the mirror angle for maximum first-pass X3 absorption on TCV [29], control of the sawtooth period by changing the EC deposition location on TCV [30], optimization of Lower Hybrid wave absporption on the FTU tokamak [31, 32, and for simulation studies of optimal plasma profile control on DIII-D [33]. Its attractiveness stems from the relative simplicity of the feedback scheme and the well-established analysis and design methods. Theoretical results proving stability of the method exist 34. The next section will outline the principles of these controllers, and show the design parameters chosen for the application on the LHD polarizers.

\subsection{Principles of extremum seeking controllers}

We will now briefly explain the basic concept of an extremum seeking controller. For a more exhaustive treatment, we refer to [34].

The extremum seeking controller used in this application is schematically displayed in Figure 2, Its working can be decomposed as follows:

- A periodic perturbation $s(t)$ of frequency $\omega_{s}$ and amplitude $a_{s}$ is injected into the plant

- The estimate $r(t)$ of the plant's response to the perturbation is passed through a high pass filter with transfer function $H(s)$ and cutoff frequency $\omega_{h}$ which removes the DC and low frequency components.

$\ddagger$ We use the term "plant", which is commonly used in the control literature, to refer to the system being controlled including sensors and actuators, in this case LHD including the polarizer system and the relevant diagnostics. 


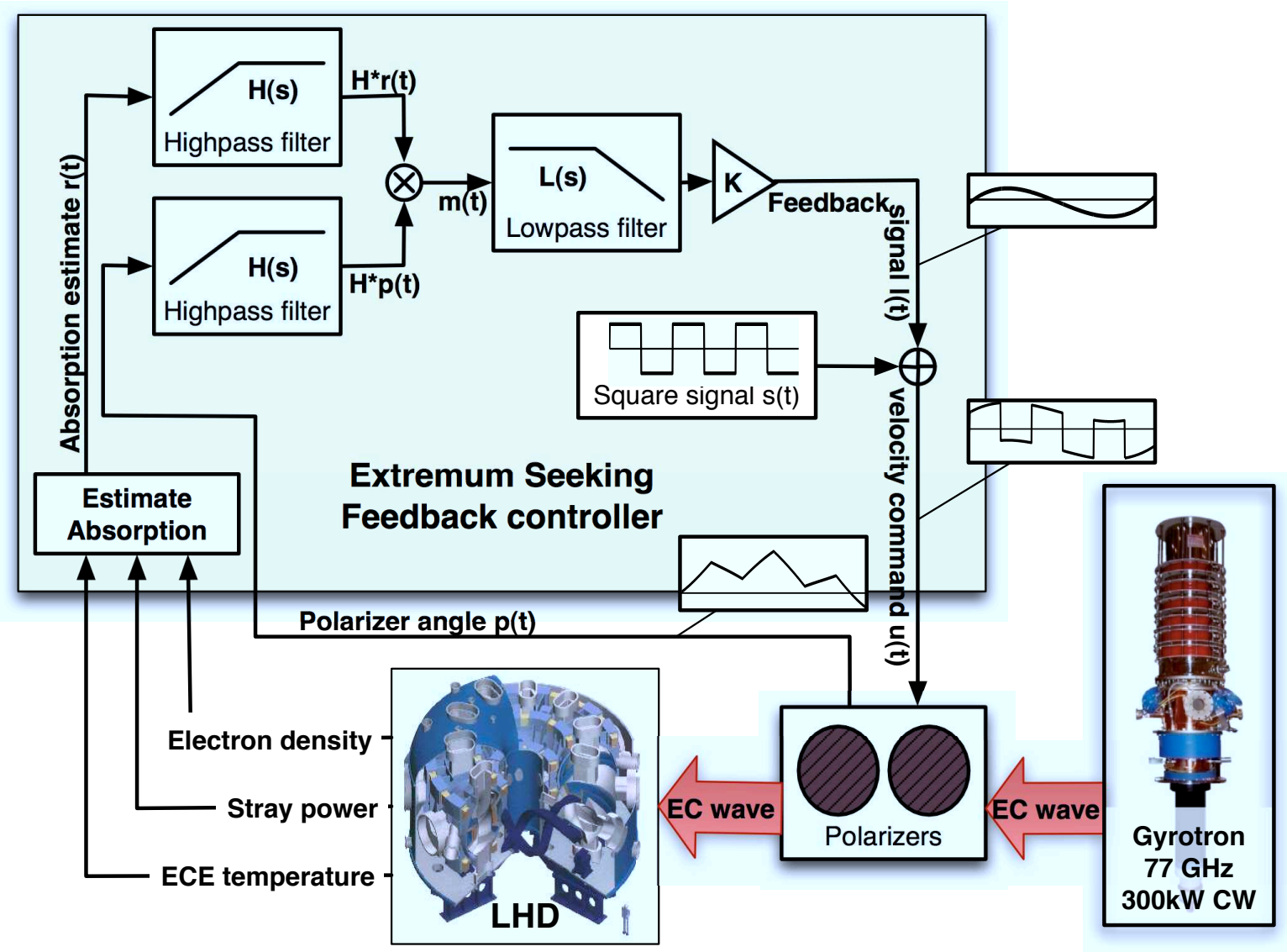

Figure 2: Schematic diagram of an extremum seeking feedback controller for the LHD polarizers. Illustrative examples of typical signals waveforms for $\ell(t), s(t), u(t)$ and $p(t)$ are shown.

- The actuator's response $p(t)$ to the periodic control signal is also measured, and passed through the same high pass filter $H(s)$.

- The two filtered signals are modulated (multiplied), giving the signal $m(t)$, in effect providing a measure of the instantaneous correlation between the two signals.

- The modulated signal is passed through a low-pass filter with transfer function $L(s)$ and cutoff frequency $\omega_{l}$. This gives the DC component of the correlation between the actuator position and the plant output.

- This signal is amplified by a feedback gain $K$ and added to the plant perturbation signal, the resulting control signal $u(t)$ is fed to the actuator.

In summary, the extremum seeking controller equation is given by the equations

$$
\begin{aligned}
& s(t)=a_{s} \operatorname{sign}\left(\sin \left(\omega_{s} t\right)\right) \quad \text { Square perturbation signal } \\
& m(t)=(H(s)[r(t)]) *(H(s)[p(t)]) \quad \text { Modulated signal } \\
& \ell(t)=K L(s)[m(t)] \quad \text { Feedback correction signal }
\end{aligned}
$$




$$
u(t)=s(t)+\ell(t) \quad \text { Velocity command }
$$

where $H(s)$ and $L(s)$ represent the transfer functions of the filters in the Laplace domain, which can be of first-order

$$
L(s)=\frac{\omega_{l}}{s+\omega_{l}}, \quad H(s)=\frac{s}{s+\omega_{h}}
$$

or can be implemented as higher order filters.

The crux of the extremum seeking scheme is that, under certain assumptions which will be mentioned below, the signal coming out of the low pass filter actually gives an estimate of the local gradient of the mapping between actuator position and plant output. If the plant output increases when the actuator position increases, the modulated signal will be predominantly positive and the lowpass filter will output a positive signal. If the plant output decreases when the actuator position increases, the modulated signal will be predominantly negative and the lowpass filter will output a negative signal. When a local maximum is reached, the plant output decreases upon both positive and negative actuator position variations. This will result in a frequency doubling which will be rejected by the low pass filter, yielding effectively zero output. By adding the resulting estimate of the positive gradient direction to the perturbation signal $s(t)$, we ensure that the controller moves the actuators towards the direction of increasing objective function, i.e. maximizing the output.

To obtain the desired behaviour, the parameters of the extremum seeking controller should be designed appropriately. It is possible to simplify the design by choosing $\omega_{l}=\omega_{h}$. The high pass filter frequencies $\omega_{h}$, should be chosen smaller than the plant perturbation frequency $\omega_{s}$ by a certain factor, which is a design parameter. The overall time scales of the extremum seeking controller are however dictated by a time scale hierarchy inherent to the control method.

Plant time scale The fastest time scale, this is the time required for the plant to respond to changes in the actuator position. If the observations are based on global plasma parameters then this is of the order of the confinement time of LHD plasmas, and the time scale is $<0.1$ s. If more direct diagnostics are used this time may be shorter.

Perturbation time scale This intermediate time scale corresponds to the typical frequency of the perturbation signal. Since the polarizers used for these experiments had a maximum rotation speed of $1 \%$, and changes to the coupling to the plasma start to become visible after $>4^{\circ}$, the typical time required for a significant perturbation is about $>4 \mathrm{~s}$.

Controller adaptation time scale The slowest time scale corresponds to the adaptation of the controller to the maximum, i.e. longer than the typical time scale of the low pass filter. This corresponds for our application to about $>40$ s.

Although the perturbation time scale could in principle be chosen shorter, it was limited in our case by the speed of the polarizers and the necessity to move at least several degrees to see an appreciable change in the plasma response. 
A very attractive feature of the extremum seeking controller is that it is readily adaptable to multivariable plants [35]. In particular, for two actuators the design does not change, as it is sufficient to make the perturbation signals orthogonal (i.e. having a $\pi / 2$ phase difference) for each polarizer. The controller signals $s(t), m(t), l(t)$ and $u(t)$ then become two-dimensional vectors. For more than two actuators, parallel feedback loops with different perturbation frequencies can be employed. Although this is not our case, we mention this property for possible future uses involving simultaneous optimization of polarizer angles and launcher angles, for example. One could also envisage the application of this technique to the multi-beam ITER ECH system, where the polarizers can be optimized one pair at a time using a fixed perturbation frequency, or multiple pairs of polarizers can be optimized at the same time using a different perturbation frequency for each pair.

\subsection{Simulations and controller parameter selection}

To choose the parameters of the controller and to test its behaviour, a series of simulations was carried out using the Matlab/Simulink software package. In these simulations, both the polarizers and the plasma response to the changing polarization were described by simplified models. The polarizers were modeled by a one-pole firstorder transfer function with response time $\tau_{p}=0.1 \mathrm{~s}$, plus a time delay accounting for signal transmission delays $\delta=0.05 \mathrm{~s}$. These model parameters were extracted from measured polarizer angle responses to command signals. The LHD first-pass absorption to the polarizer settings was modeled simply by a 2-dimensional function describing the power coupling of a polarized wave (with $\alpha, \beta$ calculated from a given $\phi_{\lambda / 8}, \phi_{\lambda / 4}$ ) to a receiver accepting only linear polarization using the equation

$$
P=\cos ^{2}\left(\alpha_{s}-\alpha\right) \cos ^{2}\left(\beta_{s}-\beta\right)+\sin ^{2}\left(\alpha_{s}-\alpha\right) \sin ^{2}\left(\beta_{s}+\beta\right)
$$

or, equivalently, $P=\cos ^{2}\left(\beta_{s}-\beta\right)-\sin ^{2}\left(\alpha_{s}-\alpha\right) \cos \left(2 \beta_{s}\right) \cos (2 \beta)$, where $\left(\alpha_{s}, \beta_{s}\right)$ correspond to the polarization accepted by the receiver, in this case $\beta_{s}=0$. The plasma response due to changing first-pass absorption is modeled as a first-order transfer function with time constant $\tau=100 \mathrm{~ms}$ to simulate the effect of the energy confinement time. White noise is artificially added to the measurements to simulate the effect of the inevitable noise present in the diagnostic signals.

The controller was simulated as a discrete-time system with finite sampling time, thus including the effects of sampling and digital filter implementation. Parameter studies resulted in the following choice for the design parameters: $f_{s}=\omega_{s} /(2 \pi)=$ $0.25 \mathrm{~Hz}, a_{s}=1^{\circ} / \mathrm{s}, \omega_{h}=0.6 \omega_{s}, K=500$. The filters were implemented as fourth-order discrete-time Butterworth filters. A square perturbation signal is chosen as this has been shown to have faster convergence [36]. Due to the low speeds involved in the controller, a controller sampling rate of $5 \mathrm{~Hz}$ was found appropriate, this being sufficiently faster than the slowest time scales involved in the control cycle $(\sim 1 \mathrm{~s})$ while being compatible with the available hardware.

$\S$ http://www.mathworks.com 
Figure 3 shows the result of a set of simulations to test the response of the extremum seeking algorithm, with the above parameters, to changes in the plasma response amplitude (corresponding to $r(t)$ in Figure 2). In this case the algorithm begins in a non-ideal configuration corresponding to $73 \%$ O-mode purity. At $t=0$, the feedback loop is closed. Within several tens of seconds the feedback loop obtains the $100 \%$ Omode purity configuration. The sensitivity of the response is investigated depending on scaling of the plasma response amplitude, for a given value of the feedback gain $K$. The response is scaled by a factor of 0.1-10. As can be seen from the figure, for the lowest response of $1 / 10$ of the nominal value, the polarizer movement is slow and dominated by the oscillatory perturbation signal. For a response of 10 times the nominal value, the algorithm is faster but more unstable, being excessively sensitive to noise and showing significant deviations from the optimal configuration.

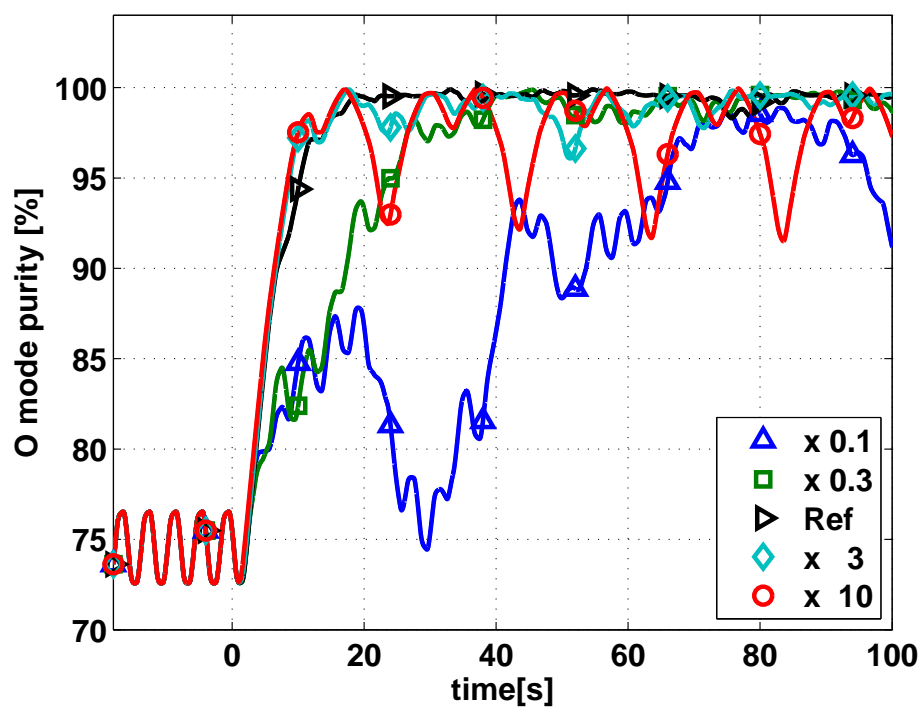

Figure 3: Robustness of the extremum seeking controller behaviour to scaling of the plasma response. For very low responses $(0.1 \times$ the reference) the controller has difficulty finding the 100\% O-mode position. For 10× higher response than expected, the behaviour is faster but more unstable, with tendency to overshoot the target polarizer rotation angles or temporarily move away. The feedback gain used for this simulation is the design value $K=500$.

\subsection{Diagnostics for measuring the EC absorption}

One of the most important design choices is the choice of the signal(s) used for estimating the first-pass absorbed power. Clearly, the degree of absorption that can be obtained by any control system is limited by the fraction of non-absorbed power that can be measured and detected. Note that for the extremum seeking scheme it is not necessary to measure the absolute value of the absorbed power, but it is sufficient to have a diagnostic which 
measures changes of absorption as accurately as possible. We discuss here a number of possible diagnostic setups, stressing the fact that the actual implementation may differ for practical reasons.

The most straightforward choice is to use a measure of the plasma temperature or internal energy, for example the product between the ECE temperature and the electron density, or a DML (diamagnetic loop) signal. One can also choose to directly measure the non-absorbed power using infrared cameras or thermocouples to detect local heating of in-vessel components. Another possibility is the use of one or multiple sniffer probe signals measuring the amount of stray power exiting the vessel [37]. If a measure of plasma energy is used, this can be affected by internal plasma processes not related to the ECRH absorption. This will be seen as a disturbance in the control system which, unless handled explicitly, can have a temporary effect on the polarizer positions depending on the time scale of the internal plasma event. Large ELMs (Edge Localised

Modes) could play this role in tokamaks. Sniffer probes provide a direct and immediate measure of the non-absorbed microwave power, but the signal level depends critically on the placement of the probes with respect to the plasma and the non-absorbed EC wave. For example, if the wave is reflected by a cutoff, as for example with X- mode in ITER at full field, the propagation direction of the resulting non-absorbed beam depends on the location of the cutoff layer. Changes in the measured signals can therefore not only be attributed to changes in the absorption, but also to density fluctuations. To get a reliable measure it may be necessary to optimize the number and placement of the stray power detectors to be independent of these factors. Alternatively, when the launching and plasma geometry are relatively fixed, optimal sniffer probe locations can be chosen to enhance the sensitivity to the undesired launch polarization.

\section{Experimental setup}

\subsection{Plasma and ECRH scenario}

The controller described in the previous section was implemented and tested during longpulse ECRH sustained plasma experiments on LHD. In these experiments, a plasma was sustained using three $77 \mathrm{GHz}$ gyrotrons for several minutes. The plasma density was of the order $0.5 \times 10^{19} \mathrm{~m}^{-3}$. The transmission lines for these gyrotrons are equipped with real-time steerable polarizers. For these experiments, we controlled the polarizers for the gyrotron connected to an outboard port providing low-field side injection. In the scenario used $\left(B_{T}=2.75 \mathrm{~T}, R_{a x}=3.75 \mathrm{~m}\right)$ the EC first harmonic resonance is located on axis, directly in front of the steering antenna. The optimum setting of the polarization has been the topic of experimental investigation in [18]. In that work, experimental observations of the ECRH absorption as a function of the polarizer settings are compared to calculations of the optimum polarization. It was shown that different choices of the location of the vacuum-plasma boundary (LCFS, Separatrix or SOL) change the calculated O-mode purity by approximately 10\%, leading to different optimum polarizer 
settings, with the SOL giving the best fit to experimental data. This choice leads to $(\alpha, \beta)=\left(45^{\circ}, 0^{\circ}\right)$, or purely linear polarization, as the optimum configuration setting corresponding to the magnetic field direction of $45^{\circ}$ at the SOL. It should be noted that a more complete calculation shown in [21] confirms this choice.

\subsection{Diagnostics}

For the experiments described in this paper, several diagnostic signals were available to the real-time controller, including two ECE channels $\left(T_{\text {ece }}\right)$ measuring the ECE temperature on the inboard side at $\rho=0.66$ and $\rho=0.73$ (no real-time measurement of a central chord was available for this magnetic configuration due to technical reasons), a central interferometer channel $\left(n_{e} L\right)$, one stray power signal $P_{\text {sniffer }}$ from a sniffer probe located in the same sector as the injected ECRH beam, and the gyrotron power $P_{\mathrm{EC}}$. In the results shown below, however, only the ECE temperature $T_{e c e}$ is used as a measure of the first-pass absorption. This was adequate since the gyrotron power and density did not change significantly. Although, as discussed in the previous section, it could be advantageous to use sniffer probe signals as a direct measure of the non-absorbed power, the sniffer probes currently installed on LHD have a low signal/noise ratio and would require further development to be used for these experiments.

\subsection{Controller implementation}

Because of the slow nature of the control cycle, it was not necessary to implement it on high-performance dedicated hardware or software. The software was developed using National Instrument: $\llbracket$ LabWindows/CVI 9.1 software running on a desktop PC with Windows XP. Cycle times were monitored to ensure that the software maintained the required $5 \mathrm{~Hz}$ frequency. The signals listed above were acquired using a NI-6218 USB device. The polarizer motor commands were transmitted to a Newport ESP300 motor controller in the RF heating room, about thirty meters away from the control computer, via an extended GPIB (IEEE 488, General Purpose Interface Bus) network. The motor controller itself commands the two rotating stages $(\lambda / 8$ and $\lambda / 4)$ originally designed for $84 \mathrm{GHz}$, acquired from General Atomics, San Diego, installed in the transmission line described above. Although originally designed for a slightly different frequency, the polarizing effect of the polarizers is still well described by a model taking the frequency difference into account.

\section{Results}

As described in section 3.1, the polarizer setting on LHD is determined prior to the discharge based on the magnetic field orientation at the SOL. This setting is referred to as the pre-determined setting, which may or may not be optimal for maximizing the first-pass absorption. In order to test the controller, we moved the polarizer angles 
during the pulse to a position away from the pre-determined setting. The feedback controller was then activated and carries out the extremum seeking, i.e. it searches for a polarizer setting which maximizes the measured ECE temperature. It was found that the algorithm returns the polarizers to their original positions, corresponding to the pre-calculated positions based on the $B$-field at the SOL. At the same time, the ECE temperature is shown to increase. This experiment was repeated for several different initial conditions with different values of the feedback gain, as shown in Figures 4 and 5 .

\subsection{Polarizer time trajectories}

Figure 4 shows the trajectories followed by the polarizers during the feedback control experiment. The traces are overlaid on the landscape portraying the O-mode purity as calculated from the SOL-based calculations. The fact that, in all three cases, the feedback control system moves the polarizers to a zone of high O-mode purity indicates the extremum seeking algorithm works well, that these calculations are indeed correct and the pre-calculated polarizer settings would have been adequate for the injection scenario considered. The spread of the established optimal setting for the polarizers is limited by the continuing movement of the polarizers in response to the feedforward perturbation signal $s(t)$, and is estimated as $5^{\circ}$. A more advanced control scheme could reduce the amplitude of the perturbation once an optimum has been reached, but will ultimately be limited by the achievable diagnostic accuracy in determining the determination of the first-pass absorption.

Figure 5 shows more in detail some of the signals which play a role in the controller. The polarizer angle traces show that the movement is a superposition of the triangular position oscillation (from a square velocity perturbation) and a slower compensation signal from the feedback loop. The lower plots show these correction signals for both polarizers, corresponding to the signal $\ell(t)$ in equation (3), as well as the high pass filtered ECE signal $H * r(t)$. The plot illustrates how the signal $\ell(t)$ is computed by lowpass filtering the noisy higher-frequency ECE oscillations to extract a correlation between the polarizer movement direction and the ECE radiation temperature variation. Figures $5 \mathrm{a}$ and $5 \mathrm{~b}$ represent two different choices of the feedback gain $K=100$ and $K=200$, respectively. In the second case, the polarizer movements are dominated less by the oscillatory perturbation signal and more by the feedback signal.

Also, in figure 6] we show the results of an experiment done in a different scenario $\left(B_{T}=2.705 \mathrm{~T}, R_{a x}=3.6 \mathrm{~m}\right)$ for which the optimum ECRH injection geometry is that of oblique injection. The EC wave polarization at the interface is then set to fully circular.

The consequence of this scenario is that the theoretical optimum is broad and depends very little on the $\lambda / 4$ angle (because if $\beta=45^{\circ}$ equation (6) becomes insensitive to $\alpha$ ). Therefore, only the $\lambda / 8$ polarizer angle was optimized in this experiment. Interestingly, during this experiment the ECE temperature was decreasing because of a slow timescale increase in plasma density; yet, since there is a correlation between the polarizer 


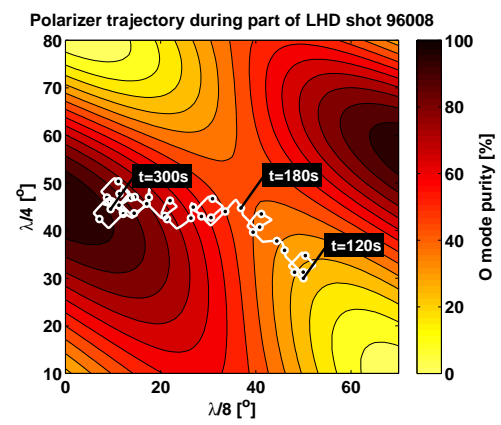

(a)

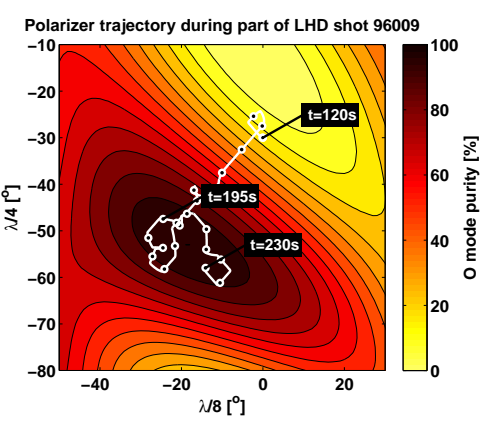

(b)

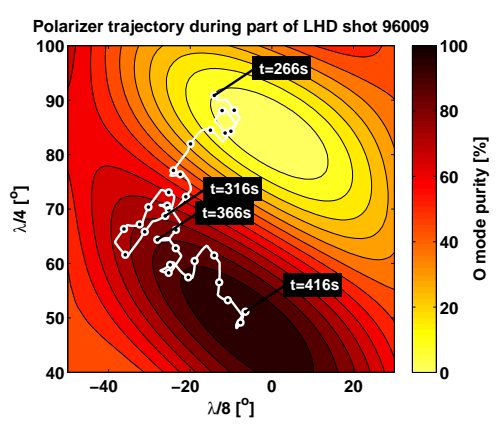

(c)

Figure 4: Evolution of the polarizer angles steered by the feedback control system. The contours indicate the O-mode purity as calculated from the knowledge of the magnetic field at the edge of the scrape-off layer. In the three cases shown, the polarizer setting was purposely brought away from the optimal setting after which the feedback controller was switched on. In all cases, the real-time control algorithm is seen to recover the optimal control settings in about 150 s.

movements and the ECE response on shorter time scales, the polarizer moves in the expected direction.

\subsection{Estimate of the obtained mode purity}

In part of shot \#96009 shown in Figure 4b, the polarizers trajectory finds the optimum position and subsequently (after $t>195 \mathrm{~s}$ ) dwells in the vicinity of this optimum for some time. This phase of the experiment allows us to estimate the precision of the feedback system and the expected mode purity. We see that the controller maintains the polarizer setting within the area of more than $90 \%$ O-mode purity. This is the maximum accuracy which can be reached with this system. It is limited by both the continuing oscillatory perturbation of the position (which, as mentioned, can be decreased using a more complex feedback scheme) but also by the diagnostic sensitivity and signal noise. With improvements, it is expected that this accuracy can be increased significantly.

\section{Discussion of the results}

The experimental results shown above demonstrate that the extremum seeking algorithm is able to find, from a non-optimal setting of the polarizer angles, a better setting corresponding to the theoretically expected optimum. Several experimental issues have to be mentioned which have affected the results. First of all, in the simulations shown in section 2.2 , the calculated O-mode purity was taken to be directly proportional to the absorption estimate. In reality this will not be the case because a) the first-pass O-mode absorption is not $100 \%$, particularly in the relatively low density plasmas obtained here and b) part of the EC power in X-mode may ultimately be 


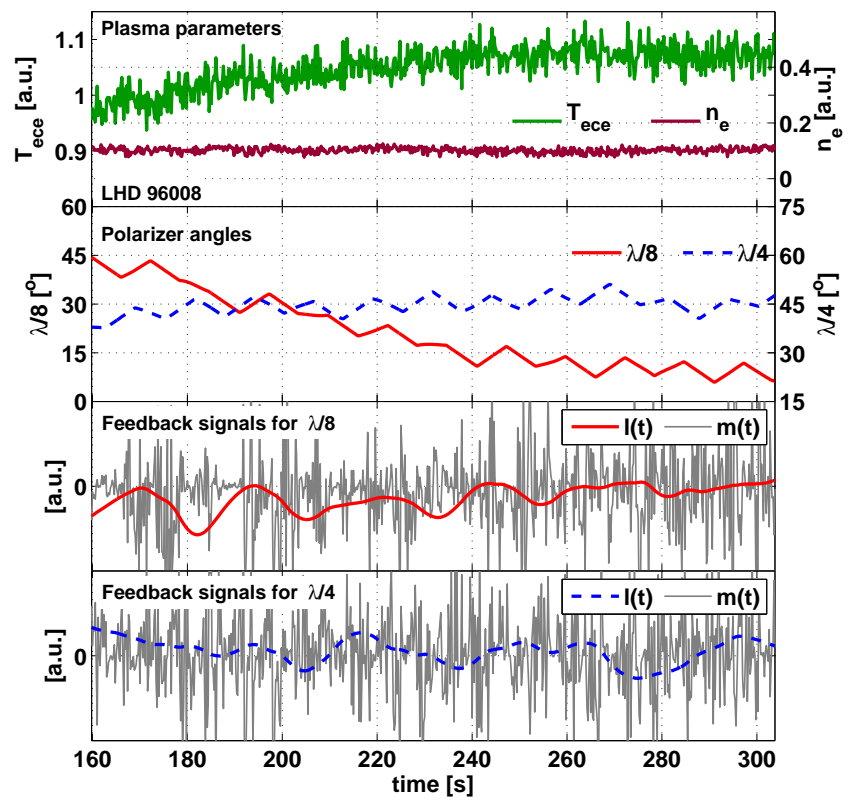

(a) Time trace corresponding to Fig 4a, $K=100$

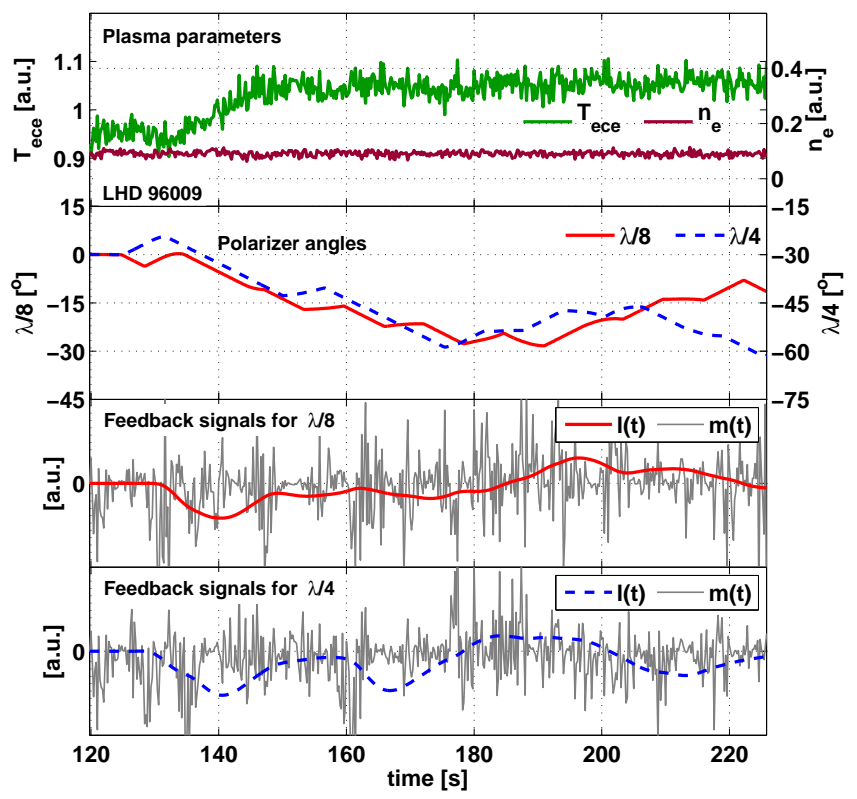

(b) Time trace corresponding to Fig $4 \mathrm{~b}, K=200$

Figure 5: Time traces corresponding to figures $4 \mathrm{a}$ and $4 \mathrm{~b}$, In the case shown in (a), the feedback gain was low $(K=100)$, compared to the design value $(K=500$, used in the simulations shown in figure (3) so the convergence to the optimum is slow and the oscillatory perturbations dominate the polarizer movement. In the case shown in (b), the gain was higher $(K=200)$ and the response is seen to be faster. The feedback signals $\ell(t)$ (equation (3) ) for each of the two polarizers can be seen in the lower time traces, indicating how the extremum seeking scheme adapts the polarizer positions corresponding to higher ECE signals. The signal $m(t)$, representing the product of the high-pass filtered ECE signal and the corresponding polarizer position is also displayed, giving a feel for the noisy signal from which the correlation information is extracted. 


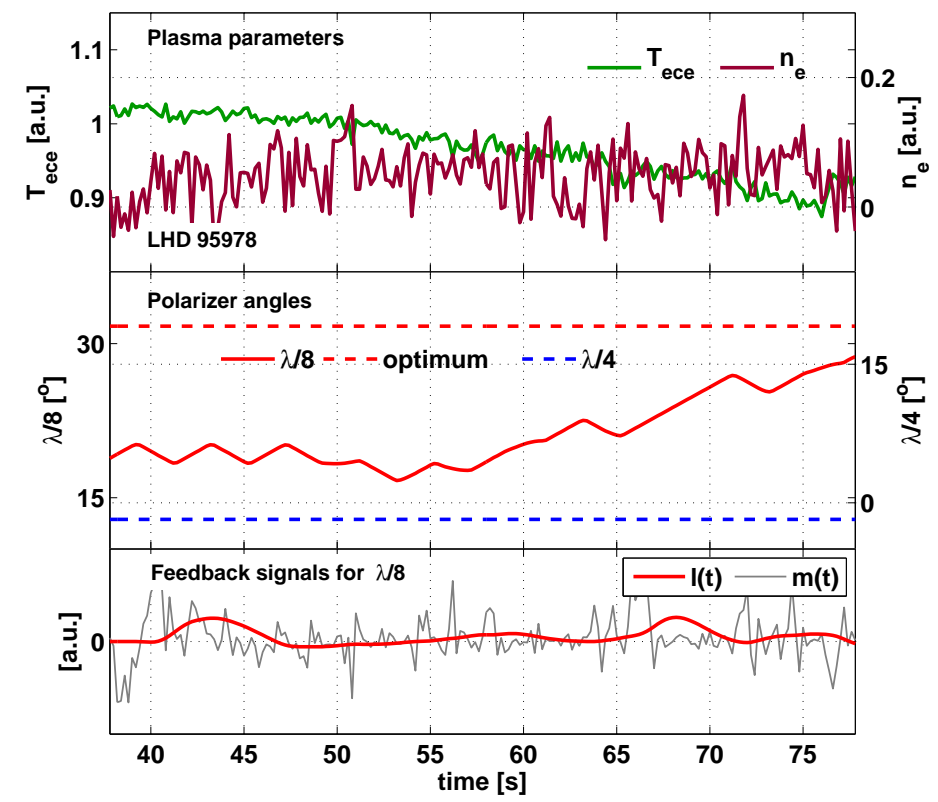

Figure 6: Time traces for demonstration of $\lambda / 8$ polarizer optimization. The extremum seeking algorithm moves the polarizer towards the direction of the pre-calculated optimal position giving fully circular polarization $\beta=45^{\circ}$. Even though the ECE temperature is seen to decrease during this period due to a density rise, it is the shorter time-scale responses to the polarizer movements (notably at $t=55 \mathrm{~s}$ and $t=65 \mathrm{~s}$ ) which cause the polarizer to move in the correct direction, on average. The feedback loop is closed at $t=49 \mathrm{~s}$, and the feedback signal, which is already positive in the period $t=40-49 \mathrm{~s}$, is not passed to the polarizer velocity commands before this time. The shot ends at $t=77 \mathrm{~s}$.

absorbed in O-mode after multiple reflections in the complicated LHD vacuum vessel geometry. This means that the effect on the observed ECE temperature of moving the polarizers will be smaller than anticipated. Finally, the effect may be more visible on a central ECE chord rather than the off-axis chords used in this experiment. The result was that during the experiments, the mean excursion of polarizer position given by the periodic perturbation had to be chosen larger than in the simulations. While a $4 \mathrm{~s}$ perturbation period $\left(f_{s}=1 / 4 \mathrm{~Hz}\right.$, yielding $\pm 1^{\circ}$ polarizer angle excursion $)$ was sufficient in the simulation, it was found that a $12 \mathrm{~s}$ perturbation $\left(f_{s}=1 / 12 \mathrm{~Hz}\right.$, yielding a $\pm 3^{\circ}$ polarizer excursion) was necessary in the experimental setting. Correspondingly, the feedback gain had to be reduced to a value of approximately $K=200$ (from the design value of $K=500$ ) to avoid overshoots and excessive polarizer movements.

Even though further optimization and parameter tuning are possible, the implemented extremum seeking controller has shown satisfactory performance. A particularly interesting case shown in figure 6, deserves more attention as it illustrates an important feature of the extremum seeking algorithm: while it is designed to adapt 
to slow scale plasma variations which affect the optimum location, it is insensitive to slow time scale variations as long as they do not affect the optimum angle settings. On the shorter time scale, the system will only react to effects on the same time scale as the local modulated polarizer movement. This implies, however, that events on time scales comparable to the polarizer oscillation time, such as disruptions, sudden density changes etc. can have a significant effect on the control system. This effect will be temporary, and if the disturbances are not too frequent the polarizers will return to their optimal position. In the experiments shown, efforts were made to minimize these disturbances by creating a plasma with stationary characteristics. Taking known disturbances into account explicitly may be possible, but will add a layer of complexity to the system.

In all the experiments, the polarization setting found by the feedback control system matched the pre-calculated value within the variance of the system. Unexpected effects of changing mode coupling due to shear effects which were one of the motivations for the feedback control system development, were not observed during these shots. In any case, the feedback controller, tested on LHD, can in principle also be used to track changes in the plasma conditions such as changes in density or plasma current (in the case of a tokamak), or variations of the injection angle. As long as sufficient time is given for the controller to act and the controller parameters are tuned appropriately, a local optimum will be found.

It should be noted that the speed of the feedback controller is limited at present by the rotation speed of the polarizers, which is $\sim 1^{\circ} / \mathrm{s}$. With faster polarizers such as those proposed for ITER [38] this speed can be increased until the velocity perturbation period is decreased to the confinement time scale. At these time scales we can however no longer consider the plasma response as approximately a static map but the plasma dynamic response will have to be taken into account. Extremum seeking controller design methods for plants described by a static map concatenated with a linear time invariant dynamical system could be used in this context [34], [35].

\section{Conclusions}

This paper has presented the design and implementation of a feedback controller for on-line optimization of the polarizer rotation angles for maximum first-pass absorption of ECRH in LHD plasmas. The experimental results have shown that

- An extremum seeking algorithm is adequately able to find the local optimum from a variety of initially non-optimal absorption settings

- The pre-calculated polarization settings, based on the knowledge of $B$ field at the edge of the scrape-off layer and the injection geometry, were adequate in the scenario tested. No better setting could be found within experimental error margins $(\geq 90 \%$ O-mode purity corresponding to a polarizer uncertainty of $\sim \pm 10^{\circ}$ ).

- As long as the plasma density and injected gyrotron power remain constant during the plasma as they did in the experiments shown here, the ECE temperature 
provides an adequate indication of the first-pass absorption of the fundamental O-mode in the plasma center for the proof-of-principle experiments described in this paper. If the maximum non-absorbed power is to be reduced below $10 \%$, a more accurate diagnostic method is required to measure the stray power.

Further extensions and improvement of the algorithm are possible and have been discussed. We also indicated the possibility of further parameter tuning to minimize the time taken for the feedback loop to reach an optimum. The algorithm can be tested against disturbances in situations where changes to the plasma cause a need for change in the polarizer settings. Finally, implementation with rapid polarizers would open the possibility of performing the optimization in a shorter time span suitable for intermediate-duration pulses and of responding more rapidly to changes in the plasma. An important point is that the method presented here is sufficiently general to be easily implemented on other tokamaks or stellarators with ECRH and controllable polarizers.

\section{Acknowledgments}

This work is supported by the National Institute for Fusion Science under grants ULRR501, 512, by NIFS/NINS under the project of Formation of International Network for Scientific Collaborations, and in part by the Swiss National Science Foundation.

\section{References}

[1] Erckmann, V. 2009 Fusion Engineering and Design 84131

[2] Iiyoshi, A. et al. 1999 Fusion Engineering and Design 46323

[3] Shimozuma, T. et al. 2009 Journal of Microwave Power \&3 Electromagnetic Energy 4343

[4] Yoshimura, Y. et al. 2005 Journal of Physics: Conference Series 25189

[5] Igami, H. et al. 2009 Nuclear Fusion 49115005 (11pp)

[6] Shimozuma, T. et al. 2008 Journal of Physics: Conference Series 123012022 (9pp)

[7] Takahashi, H. et al. 2010 Fusion Science and Technology 5719

[8] Erckmann, V. et al. 2005 in AIP Conference Proc. of $16^{\text {th }}$ Conference on RF Power in Plasmas volume 787 Park City, USA 371-378

[9] Laqua, H. et al. 2007 Fusion Engineering and Design 82982

[10] Henderson, M. et al. 2008 Nuclear Fusion 48054013 (14pp)

[11] Takahashi, K. et al. 2008 Nuclear Fusion 48054014 (9pp)

[12] Prater, R. 2004 Physics of Plasmas 112349

[13] Stix, T. 1992 Waves in plasmas Springer, New York

[14] Smits, F. 1989 A polarising mirror for electron cyclotron resonance heating on RTP Ph.D. thesis Rijnhuizen report 91-208 FOM, The Netherlands

[15] Doane, J.L. 1992 International Journal of Infrared and Millimeter Waves 131727

[16] Thumm, M. et al. 2002 IEEE Transactions on Plasma Science 30755

[17] Wagner, D. et al. 2005 International Journal of Infrared and Millimeter Waves 26163

[18] Notake, T. et al. 2005 Plasma Physics and Controlled Fusion 47531

[19] Morisaki, T. et al. 2003 Journal of Nuclear Materials 313-316 548

[20] Fidone, I. et al. 1971 Nuclear Fusion 11133

[21] Segre, S.E. et al. 2006 Plasma Physics and Controlled Fusion 48599

[22] Bigelow, T. 1990 A High-Power Microwave Transmission and Launching System for Plasma Heating on the ORNL ATF Experiment. Ph.D. thesis University of Tennessee 
[23] Nagasaki, K. et al. 1999 Physics of Plasmas 6556

[24] Yoshimura, Y. et al. 2006 Journal of the Physical Society of Japan 75114501

[25] Idei, H. et al. 2001 Fusion Engineering and Design 53329

[26] de Vries, P.C. et al. 2000 Physics of Plasmas 73707

[27] Press, W.H. et al. 1996 Numerical Recipes Cambridge University Press, New York second edition

[28] Felici, F. et al. 2009 Review of Scientific Instruments 80013504

[29] Alberti, S. et al. 2005 Nuclear Fusion 451224

[30] Paley, J.I. et al. 2009 Plasma Physics and Controlled Fusion 51124041 (11pp)

[31] Centioli, C. et al. 2008 Control Engineering Practice 161468

[32] Carnevale, D. et al. 2009 Fusion Engineering and Design 84554

[33] Ou, Y. et al. 2008 Plasma Physics and Controlled Fusion 50115001 (24pp)

[34] Krstic, M. et al. 2000 Automatica 36595

[35] Ariyur, K. et al. 2002 in Proceedings of the 2002 American Control Conference volume 4 Anchorage, AK 2903-2908

[36] Tan, Y. et al. 2008 Automatica 441446

[37] Gandini, F. et al. 2001 Fusion Engineering and Design 56-57 975

[38] Doane, J. et al. 2008 Fusion Science and Technology 5339 